\title{
Quimioluminiscencia como método de screening de cáncer oral
}

\section{Chemiluminescence as a screening method in oral cancer}

\section{Carrera Torres A*, Cobos Fuentes MJ*, Gallardo Castillo I**, Caballero Aguilar J**, Martínez-Sahuquillo Márquez A***}

\section{RESUMEN}

El cáncer oral es un problema de salud con el que nos enfrentamos todos los días en nuestras consultas. Los dentistas formamos la primera línea de defensa ante una patología que no ha presentado una mejora de los índices de mortalidad y morbilidad en las últimas décadas. La principal arma con la que contamos es la del diagnóstico precoz de la enfermedad, y esto es mucho más complicado de lo que parece. Para ayudar a los dentistas en la visualización de lesiones de precáncer o cáncer temprano han aparecido algunas técnicas o dispositivos de screening de cáncer oral basadas en la interacción de los tejidos orales con la luz. La técnica más extendida y estudiada es la basada en la quimioluminiscencia, intentaremos poner en claro algunos aspectos analizando los estudios que hay en la literatura actual.

Palabras clave: Screening, cáncer oral, quimioluminiscencia.

\section{SUMMARY}

Oral cancer is a health problem with which we face every day in our consultations. Dentists form the first line of defense against a disease that has not produced an improvement in mortality and morbidity in recent decades. The main weapon with which we have is the early diagnosis of the disease, and this is much more complicated than it seems. To assist dentists in the visualization of pre-cancer lesions or early cancer have appeared some techniques and devices for oral cancer screening based on interaction of the oral tissues with light. The most widely used and studied technique is based on the chemiluminescence. Above it has been much talk and try to clarify some aspects of the studies that are analyzing the current literature.

Key words: Screening, oral cancer, chemiluminescence.

Fecha de recepción: 20 de mayo de 2010.

Aceptado para publicación: 27 de mayo de 2010.

* Colaborador Clínico de Medicina Bucal. Facultad de Odontología de Sevilla.

** Profesor Asociado de Medicina Bucal. Facultad de Odontología de Sevilla.

*** Profesor Titular de Medicina Bucal. Facultad de Odontología de Sevilla.

Carrera Torres A, Cobos Fuentes MJ, Gallardo Castillo I, Caballero Aguilar J, Martínez-Sahuquillo Márquez A. Quimioluminiscencia como método de screeninig de cáncer oral. Av. Odontoestomatol 2011; 27 (6): 301 311.

\section{INTRODUCCIÓN}

En la actualidad se estima que se producen en el mundo unos 643.000 casos nuevos de cáncer de cabeza y cuello al año (1), de los que el $40 \%$ son carcinomas orales de células escamosas (COCE). El cáncer oral ocupa el sexto puesto en el número de cánceres totales (2). En la Unión Europea, España está en el segundo lugar en cuanto a incidencia de la enfermedad, sólo por detrás de Francia (3). Estu- 
dios de cohortes demuestran que se ha producido un aumento de la incidencia del cáncer oral en 19 de 24 países estudiados en la unión europea (4), encontrándose un riesgo de 3 a 10 veces mayor de padecer la enfermedad de una generación a la siguiente (5). Además, pese a los avances quirúrgicos y de tratamiento, los porcentajes de supervivencia a cinco años no han variado (6). Esto es debido al retraso que se produce en la detección de la lesión cancerosa (7). Así, según los últimos estudios, el $60 \%$ de los tumores malignos diagnosticados en la cavidad oral, lo son en el estadio III o IV (6), lo que supone que exista ya una invasión linfática y por tanto que sean necesarios unos tratamientos más agresivos y a menudo mutilantes (8). Las causas de estos diagnósticos tardíos serían el debut asintomático de esta patología en numerosas ocasiones, el desconocimiento de la enfermedad por parte de los pacientes (6) y la falta de una metodología en la detección de lesiones precancerosas o de cáncer en sus estadios más tempranos $(9,10)$. La identificación de lesiones de alto riesgo puede ser la clave para la supervivencia del paciente (7) y para disminuir la agresividad de los tratamientos (7, 10-12), y los clínicos somos los responsables de buscar e identificar estos posibles cambios en las mucosas (13).

Se denomina screening o despistaje a "la aplicación de pruebas o test en pacientes aparentemente sanos o libres de una enfermedad en cuestión para clasificar a aquellos que probablemente tengan la enfermedad de aquellos que probablemente no la tengan" (14). El factor más importante del screening es que atañe a pacientes que no tienen síntomas. No hay que confundir screening con diagnóstico, ya que este último se realiza cuando la enfermedad ya está presente y existen síntomas o lesiones. Un screening en la población general no nos asegura una disminución significativa de la mortalidad del cáncer oral (15), aunque en pacientes valorados de alto riesgo; aquellos que tienen hábitos como el tabaco y/o alcohol (16), pacientes mayores de 40 años (17) o con historia de cáncer o precáncer oral, reduciría considerablemente la morbilidad y mortalidad de la enfermedad (18-22).

La Agencia Internacional para la Investigación del Cáncer (IARC) y la OMS calculan que se pueden evitar millones de muertes en el futuro si se planean y se llevan a cabo estrategias de controles efectivos y de screening eficientes de la enfermedad (1). La estrategia más recomendable sería:

- Concienciar a la población de la utilidad de revisiones periódicas.

- Utilizar sistemas de ayuda para la detección de pequeñas lesiones iniciales de cáncer y precáncer, es decir unas pruebas adecuadas de screenning (6).

Una buena prueba de screening ha de cumplir una serie de requisitos (Tabla 1 ), ya que ha de aplicarse muchas veces y por un amplio abanico de operadores. El gold-standard para una prueba de screening de cáncer oral sería la opinión de un experto en medicina oral o en patología maxilofacial, el problema es que la primera línea de screening la ocupan los dentistas generales, por tanto necesitan herramientas que les acerquen lo más posible a ese goldstandard (6).

En la actualidad, la técnica de screening más recomendable para la detección de cáncer y precáncer oral es la exploración visual convencional bajo la luz del sillón dental (EC) $(6,23)$. El 94,1\% de los dentistas generales la utilizan (10), ya que es una técnica barata, simple y aceptable (24). Numerosos autores abogan por una exploración estándar y reproducible de los tejidos blandos de cabeza y cuello que dure

\section{TABLA 1.- CARACTERÍSTICAS IDEALES DE UINA PRUEBA DE SCREENING O DESPISTAJE}

- Ser simple, segura y aceptable por el paciente.

- Detectar la enfermedad de forma temprana, en sus estadios más iniciales.

- Detectar aquellas lesiones que son susceptibles de progresar hacia estados de malignidad.

- Detectar lesiones que sean tratables de forma temprana para impedir su progresión.

- Tener un alto valor predictivo positivo y un bajo índice de falsos negativos.

- Tener un coste/beneficio aceptable. 
entre 90 y 180 segundos como un buen método de detección de cáncer oral (25), pero también hay quién piensa que aunque se hiciera así, no sería suficiente para el diagnóstico precoz de la enfermedad (22). La EC ha sido muy exitosa como técnica de screening de cáncer en zonas accesibles como la piel (26), pero en la cavidad oral nos encontramos con una serie de características que hacen que la EC tenga algunas limitaciones (27-29) (Tabla 2). La literatura estima la sensibilidad y especificidad de la EC como adecuadas, del 74-85\% y 75-99\%, respectivamente (30). Sin embargo, algunos autores piensan que con esos valores de sensibilidad la EC no sería una buena prueba de screening dado la gravedad que supone un falso negativo en una enfermedad como el cáncer oral (6), por lo que propugnan encontrar otro tipo de pruebas de screening más sensibles.

Una de las técnicas coadyuvantes de screening de cáncer oral que ha acaparado más atención en la última época es la quimioluminiscencia y su interacción con los tejidos. La primera forma de luminiscencia artificial (quimioluminiscencia) fue ideada en 1669 por Henning Brand, el físico alemán descubridor del fósforo. Fue Eilhardt Weidemann en 1888 quien acuñó por primera vez el término quimioluminiscencia, definiéndola como la energía en forma de luz que dejan escapar dos componentes químicos al ser mezclados y provocar una reacción.

\section{TABLA 2.- PROBLEMAS DE LA EXPLORACIÓN VISUIAL CONVENCIONAL DE LA CAVIDAD ORAL}

- Entre un 5-15\% de la población tiene anormalidades en la mucosa oral que pueden ser confundidas fácilmente con lesiones cancerosas.

- La mayoría de los dentistas, mediante un examen visual, son incapaces de señalar si un parche blanco va a evolucionar a un cáncer o no.

- Hay zonas con displasias o con carcinomas "in situ" que presentan un aspecto completamente normal.

- Por las características de la cavidad oral, ésta se ve sometida a numerosos traumatismos que implican el desarrollo de lesiones reactivas fácilmente confundibles con entidades malignas.
La técnica de screening de detección de cáncer oral está basada en la forma que tienen los tejidos de reaccionar ante la luz quimioluminiscente, y está ideada a partir de una prueba similar que se realiza en la detección temprana de cáncer de cérvix de útero, llamada Especuloscopia de Barrido "Speculoscopia". En diferentes estudios, esta técnica ha demostrado un valor predictivo negativo (VPN) del 94,7 al 99\% $(31,32)$ y un valor positivo coste-beneficio respecto a la exploración convencional (33). El sistema se basa en que una luz blanca-azul es absorbida por las células de los tejidos normales y reflejada por las células con núcleos anormales como las que se encuentran en tejidos displásicos o neoplásicos. Previamente los tejidos han de ser sometidos a una solución de ácido acético al $1 \%$ que, aplicada durante cierto tiempo en forma de enjuague, elimina posibles residuos epiteliales, destruye temporalmente la barrera de glicoproteínas de la superficie de la mucosa y provoca una deshidratación citoplasmática, dejando a la luz penetrar y provocando un cambio en las propiedades refractarias de los tejidos (34).

Tras la aplicación de la solución deshidratante, y bajo la luz quimioluminiscente, aquellos tejidos que contengan células con un ratio núcleo-citoplasma alterado y tejidos hiperqueratinizados o con cambios inflamatorios presentarán un aspecto "acetoblanco" que es como se definiría al aspecto blanquecinodeshidratado y más opaco de lo normal (35).

La Administración de Estados Unidos de Norteamérica de Alimentos y Medicamentos (U.S.F.D.A.) tiene establecido como una de las aplicaciones de la técnica de luz quimioluminiscente la detección de lesiones cancerosas, tanto en el campo de la ginecología como en la mucosa oral (22), y en 2004 la Asociación Dental Americana (ADA) ofrecía un reembolso parcial por la compra de dispositivos basados en la quimioluminiscencia (36).

El único sistema comercializado está compuesto por un enjuague de ácido acético al 1\% (propileno glicol + alcohol + benzoato de sodio + ácido acético al $1 \%+$ aromotizantes de frambuesa) y un sistema de luz quimioluminiscente (un vial que contiene peróxido de hidrógeno + ácido acetilsalicílico que ha de romperse para activarse) que emite una luz de longitud de onda de 430 a $580 \mathrm{~nm}$. durante 10 minutos. 
Además lleva añadido un dispositivo para realizar una tinción con azul de toluidina, para disminuir el número de falsos positivos al aplicarlo a lesiones encontradas con el método de quimioluminiscencia que muestren cierto grado de sospecha. El uso del sistema requiere seguir un protocolo se manejo (Tabla 3). Es una técnica fácil, segura, no invasiva, bien aceptada por los pacientes y sin efectos secundarios (37).

\section{TABLA 3.- PROTOCOLO DE UTILIZACIÓN DEL SISTEMA DE QUIMIOLUMINISCENCIA}

- Utilizar el dispositivo quimioluminiscente en el paciente:

- Enjuague durante 30 segundos con la solución de ácido acético al $1 \%$.

- Expulsar el enjuague sin aclarar con agua.

- Activar el dispositivo quimioluminiscente. Romper el vial interno de la cápsula para mezclar los dos componentes y así iniciar la reacción química que dará lugar a la emisión de luz.

- Examinar al paciente de manera minuciosa toda la mucosa, explorando con las luces del gabinete y del sillón dental apagadas con el fin de apreciar mejor la interacción de la luz quimioluminiscente con la mucosa.

- Si se observa alguna lesión que presente un aspecto "aceto-blanca" se considerará como positiva a la prueba.

- Valorar el grado de sospecha que tiene la lesión, realizando un diagnostico de presunción de la lesión.

- Si la lesión es considerada sospechosa se aplicará en ella el tinte azul de toluidina.

- Aplicar la torunda 1 con ácido acético al 1\% durante 30 segundos.

- Aplicar la torunda con cloruro tolonio al 0,5\% en la zona durante 60 segundos.

- Aplicar la torunda 3 con ácido acético al 1\% en la zona, con el fin de eliminar el exceso de tinte.

- Si la lesión se tiñe con el azul de toluidina será biopsiada para su posterior estudio anatomopatológico.
El objetivo de este artículo es revisar qué existe en la literatura acerca de esta técnica de screening y diagnóstico precoz, qué ventajas ofrece y cuáles son las principales carencias del método.

\section{Ensayos clínicos con un sistema de detección de cáncer oral basado en la quimioluminiscencia}

A continuación se describirán los ensayos clínicos que hay publicados hasta la fecha evaluando la técnica de quimioluminiscencia como herramienta de detección y diagnóstico de cáncer oral. Sus características se resumen en la Tabla 4.

Hubber y cols. en 2004 (38), realizaron un estudio en el que se analizaron 150 pacientes sin enfermedad cuyo criterio de inclusión era la llegada a la consulta y el consentimiento para la prueba, buscando lesiones en la mucosa oral. La mayoría de lesiones encontradas fueron de carácter benigno (leucoedema, línea alba, tatuajes, queratosis friccionales, úlceras traumáticas, etc.). De éstas, casi ninguna fue realzada al utilizarse el sistema de quimioluminiscencia, excepto el leucoedema que resultó positivo a la quimioluminiscencia en el $100 \%$ de los casos. Dos leucoplasias fueron localizadas bajo exploración visual convencional y una más con el sistema de quimioluminiscencia (la cual no había sido detectada con la exploración convencional bajo la luz del sillón). Ninguna de ellas al ser biopsiada y estudiada mostró cambios malignos. Este estudio no analiza la especificidad, sensibilidad y Valor Predictivo Negativo (VPN) o Positivo (VPP) de la prueba (Tabla 5), aunque los autores, como opinión personal, señalan que el dispositivo de quimioluminiscencia posee gran sensibilidad y muy baja especificidad.

Un año más tarde, Ram y Siar (37) compararon la exploración visual convencional con un sistema de quimioluminiscencia y con la tinción de azul de toluidina aplicada en enjuague en 40 pacientes mayores de 35 años con historia previa de lesiones malignas en la cavidad oral o con hábitos perniciosos (betel, alcohol y tabaco). Tras estas tres exploraciones, se biopsiaron todas las lesiones encontradas, considerando como gold-standard el resultado del estudio anatomopatológico de las mismas. El siste- 


\section{TABLA 4.- CARACTERÍSTICAS DE LOS ENSAYOS CLÍNICOS REALIZADOS HASTA LA FECHA CON SISTEMA DE QUIMIOLUMINISCENCIA}

\begin{tabular}{|c|c|c|c|c|c|c|c|}
\hline Autores & Observador & Pacientes & Sistema & Ventajas & Desventajas & Sensibilidad & Especificida \\
\hline $\begin{array}{l}\text { Hubber } \\
\text { y col. } \\
\text { en } 2004\end{array}$ & $\begin{array}{c}\text { Especialista } \\
\text { en medicina } \\
\text { bucal }\end{array}$ & $\begin{array}{l}\text { 150. Sin } \\
\text { criterios de } \\
\text { inclusión }\end{array}$ & Vizilite $^{\mathrm{TM}}$ & $\begin{array}{c}\text { Gran } \\
\text { sensibilidad }\end{array}$ & $\begin{array}{c}\text { Baja } \\
\text { especificidad }\end{array}$ & $\begin{array}{c}\text { No } \\
\text { determinada }\end{array}$ & $\begin{array}{c}\text { No } \\
\text { determinada }\end{array}$ \\
\hline $\begin{array}{l}\text { Ram } \\
\text { y Siar } \\
\text { en } 2005\end{array}$ & $\begin{array}{c}\text { Especialista } \\
\text { en medicina } \\
\text { bucal }\end{array}$ & $\begin{array}{l}\text { 40. Con } \\
\text { lesión } \\
\text { previa }\end{array}$ & $\begin{array}{l}\text { Vizilite } \\
\text { Plus }^{\mathrm{TM}}\end{array}$ & Gran ayuda & & $100 \%$ & $14,2 \%$ \\
\hline $\begin{array}{l}\text { Kerr } \\
\text { y cols. } \\
\text { en } 2006\end{array}$ & $\begin{array}{c}\text { Especialista } \\
\text { en medicina } \\
\text { bucal. }\end{array}$ & $\begin{array}{l}\text { 501. Mayores } \\
\text { de } 40 \text { años } \\
\text { y fumadores }\end{array}$ & Vi & \begin{tabular}{|c|} 
Mejora la \\
visualización \\
de las lesiones \\
\end{tabular} & & $\begin{array}{c}\text { No } \\
\text { determinada }\end{array}$ & $\begin{array}{c}\text { No } \\
\text { determinada }\end{array}$ \\
\hline $\begin{array}{l}\text { Epstein } \\
\text { y cols. } \\
\text { en } 2006\end{array}$ & $\begin{array}{c}\text { Especialistas } \\
\text { en medicina } \\
\text { bucal }\end{array}$ & $\begin{array}{l}\text { 134. Con } \\
\text { lesión previa }\end{array}$ & Vizilite $^{\mathrm{TM}}$ & $\begin{array}{l}\text { Las lesiones } \\
\text { blancas } \\
\text { son más } \\
\text { identificables }\end{array}$ & $\begin{array}{c}\text { No aporta } \\
\text { beneficios a } \\
\text { la exploración } \\
\text { convencional }\end{array}$ & $\begin{array}{c}\text { No } \\
\text { determinada }\end{array}$ & $\begin{array}{c}\text { No } \\
\text { determinada }\end{array}$ \\
\hline $\begin{array}{l}\text { Farah } \\
\text { y cols. } \\
\text { en } 2006\end{array}$ & $\begin{array}{c}\text { Especialista } \\
\text { en medicina } \\
\text { bucal }\end{array}$ & $\begin{array}{l}\text { 55. Con } \\
\text { lesión blanca } \\
\text { previa }\end{array}$ & Vizili & $\begin{array}{c}\text { Mejora } \\
\text { subjetiva del } \\
\text { aspecto de } \\
\text { la lesión }\end{array}$ & $\begin{array}{c}\text { No aporta } \\
\text { nada a la } \\
\text { exploración } \\
\text { convencional }\end{array}$ & $100 \%$ & $0 \%$ \\
\hline $\begin{array}{l}\text { Oh and } \\
\text { Laskin } \\
\text { en } 2007\end{array}$ & $\begin{array}{c}\text { Especialista } \\
\text { en medicina } \\
\text { bucal }\end{array}$ & $\begin{array}{l}\text { 100. Fumador } \\
\text { mayor de } \\
40 \text { años }\end{array}$ & Vizilite $^{\mathrm{TM}}$ & \begin{tabular}{|c|} 
El ácido \\
acético mejora \\
la visualización \\
de las lesiones
\end{tabular} & $\begin{array}{l}\text { La luz dificulta } \\
\text { la visualización } \\
\text { de la lesión }\end{array}$ & $\begin{array}{c}\text { No } \\
\text { determinada }\end{array}$ & $\begin{array}{c}\text { No } \\
\text { determinada }\end{array}$ \\
\hline $\begin{array}{l}\text { Epstein } \\
\text { y cols. } \\
\text { en } 2008\end{array}$ & $\begin{array}{c}\text { Especialista } \\
\text { en medicina } \\
\text { bucal }\end{array}$ & $\begin{array}{l}\text { 84. De alto } \\
\text { riesgo y con } \\
\text { lesión previa }\end{array}$ & $\begin{array}{l}\text { Vizilite } \\
\text { Plus }^{\mathrm{TM}}\end{array}$ & \begin{tabular}{|c|} 
Facilita la \\
visualización \\
de las lesiones \\
\end{tabular} & $\begin{array}{c}\text { Muchos falsos } \\
\text { positivos }\end{array}$ & $\begin{array}{c}\text { No } \\
\text { determinada }\end{array}$ & $\begin{array}{c}\text { No } \\
\text { determinada }\end{array}$ \\
\hline $\begin{array}{l}\text { Mehrotra } \\
\text { y cols. } \\
2010\end{array}$ & $\begin{array}{c}\text { Especialistas } \\
\text { en medicina } \\
\text { bucal }\end{array}$ & $\begin{array}{c}\text { 102. Con } \\
\text { lesiones ya } \\
\text { diagnosticadas }\end{array}$ & $\begin{array}{l}\text { Vizilite } \\
\text { Plus }^{\mathrm{TM}}\end{array}$ & No tiene & $\begin{array}{l}\text { Es un test muy } \\
\text { poco fiable y no } \\
\text { aporta ningún } \\
\text { beneficio }\end{array}$ & VPP 0\% & VPN $94,8 \%$ \\
\hline
\end{tabular}

ma de quimioluminiscencia mostró una sensibilidad del $100 \%$ para lesiones malignas y una especificidad del 14,2\%. Mostró además coincidir con el gold-standard en el $80,6 \%$ de los casos respecto a lesiones malignas y premalignas. El azul de toluidina en enjuague mostró una sensibilidad del $70,3 \%$ y una especificidad de $25 \%$, coincidiendo con el goldstandard en el $64,5 \%$ de los casos. Concluyen afirmando que el sistema de quimioluminiscencia puede ser de gran ayuda, pero necesita más ensayos ya que éste se ha realizado con pacientes de mucho riesgo y con lesiones en un estado de malignidad muy avanzado.

En otro ensayo, en 2006 (39), se aplicó el dispositivo de detección basado en la quimioluminiscencia sobre 501 pacientes cuyo criterio de inclusión era ser mayores de 40 años y fumadores. Se sometió a estos pacientes a una exploración visual y a la posterior exploración con el sistema de quimioluminiscencia 


\section{TABLA 5.- MEDIDAS BÁSICAS DE EXACTITUID DE PRUIEBAS DE DIAGNÓSTICO O DE SCREENING}

- Sensibilidad: Probabilidad de que alguien con la enfermedad presente, genere un resultado positivo en la prueba.

- Especificidad: Probabilidad de que alguien que no padece la enfermedad, genere un resultado negativo en la prueba.

- Valor Predictivo Positivo (VPP): Probabilidad de que alguien que dé positivo en la prueba, padezca la enfermedad.

- Valor Predictivo Negativo (VPN): Probabilidad de que alguien que dé un resultado negativo en la prueba, no tenga la enfermedad.

buscando lesiones en la mucosa oral. Hallaron 410 lesiones en 270 pacientes. Todas las lesiones fueron halladas con la exploración visual convencional bajo la luz sillón dental, y tras someterlas al sistema de quimioluminiscencia el $61 \%$ de las lesiones que los observadores consideraban "sospechosas" fueron realzadas por la prueba y el $5,8 \%$ de las consideradas como "no sospechosas". El problema es que no se realizaron biopsias, por lo que no se pudo hallar la sensibilidad y la especificidad de la prueba. Concluyen destacando que el método puede ser útil como prueba de screening, ya que es capaz de realzar determinadas lesiones haciéndolas más identificables.

Ese mismo año, Farah y cols. (34) valoraron la eficacia del sistema de luz quimioluminiscente visualizando lesiones blancas en la cavidad oral. El estudio se llevó a cabo en un centro especializado en medicina oral donde se reclutaron 55 pacientes que hubieran sido remitidos con una lesión blanca en un periodo de tres meses, éstos fueron observados por dos especialistas en medicina bucal que dotaron a las lesiones de una clasificación de 1 a 4 dependiendo de la facilidad de observación y cualidades tales como nitidez, bordes definidos, opacidad, etc... Tras lo cual, las lesiones fueron nuevamente observadas y valoradas usando el sistema de quimioluminiscencia. Además, se realizó un diagnóstico provisional y todas las lesiones fueron biopsiadas para su posterior análisis. Fueron localizadas 55 lesiones principales y 25 satélites con la inspección visual con luz del sillón dental, y con el sistema de quimioluminiscencia mejoró subjetivamente la visualización de las lesiones y se localizó una lesión satélite nueva. Pero no existieron diferencias significativas en cuanto al tamaño, facilidad de visualización y bordes de las lesiones. En cuanto al diagnóstico de las lesiones, todas aquellas que aparecieron como positivas para el sistema de quimioluminiscencia presentaron en los estudios anatomopatológicos diferentes resultados, llegando por tanto a la conclusión de que el sistema no es capaz de distinguir hiperplasia epitelial, displasia, condiciones inflamatorias o carcinoma. En el presente estudio el sistema de quimioluminiscencia presenta una especificidad del $0 \%$ y una sensibilidad del $100 \%$ para la detección de lesiones malignas y premalignas. Los autores concluyen que el dispositivo de luz química no es efectivo y no aporta ninguna ayuda ya que no es capaz de distinguir una lesión benigna de una displasia epitelial.

Epstein y cols. en 2006 (40) aplicaron el sistema de quimioluminiscencia sobre 134 pacientes cuyo criterio de inclusión fue presentar una lesión en la mucosa oral potencialmente sospechosa. Se hallaron 138 lesiones mediante exploración visual convencional, con luz del sillón dental, de las cuales el $89 \%$ fueron descritas como una placa blanca que no se desprende al raspado. Todos los pacientes fueron sometidos al sistema de luz química para valorar los cambios provocados en las lesiones (brillo, color, tamaño, textura, etc.). Casi todas las lesiones mejoraron sus propiedades visuales bajo la luz quimioluminiscente, haciéndose más fácilmente identificables. Sólo tres no se observaron mejor, correspondiéndose con dos lesiones rojas no sospechosas de malignidad y una placa blanca en encía asociada a un liquen plano oral. Además, se identificaron dos lesiones con el sistema de quimioluminiscencia que no habían sido halladas bajo la exploración convencional, las dos en pacientes con historia previa de cáncer oral. Una se correspondió con un carcinoma oral de células escamosas recurrente y otra con una queratosis benigna. Los autores no dan resultados de sensibilidad y especifici- 
dad, pero sí señalan que la prueba muestra muchos falsos positivos y ningún falso negativo. Los autores concluyen que el sistema mejora la percepción de las lesiones con un componente blanco o rojo-blanco pero que no aporta una gran ventaja a la exploración convencional.

Los mismos autores en 2008 (41) realizaron un estudio multicéntrico (en tres universidades) en el que se valoraba la utilidad del sistema de quimioluminiscencia junto a un dispositivo de azul de toluidina para aumentar la especificidad de la prueba. Se incluyeron 84 pacientes clasificados de alto riesgo (edad, hábitos perniciosos o historia previa de cáncer y/o lesiones con displasia oral) que presentaran una lesión sospechosa en una exploración visual con luz del sillón. Todas las lesiones fueron datadas y descritas, posteriormente se dataron y describieron de nuevo utilizando el sistema de luz química. A todas las lesiones que resultaron positivas a la quimioluminiscencia se les sometió a una nueva exploración con azul de toluidina, y tras esto, todas las lesiones fueron biopsiadas y sometidas a un estudio anatomopatológico por un patólogo "ciego". Las lesiones fueron caracterizadas por un mismo observador, el cual anotó sus opiniones subjetivas respecto a los cambios producidos en el aspecto de la lesión al aplicarle el ácido acético y la quimioluminiscencia: si mejora o no la percepción, si cambia, si aparecen lesiones satélites, etc. Tras aplicar el azul de toluidina también se anotó qué lesiones fijaban el tinte y además si esta fijación era completa o no.

Todas aquellas lesiones que fueron halladas por exploración visual con luz del sillón dental dieron positivo a la visualización con la quimioluminiscencia y en el $61,8 \%$ de los casos la lesión fue mejor apreciada por el observador (color, textura, brillo). Así pues, los autores señalan que un 55\% de las lesiones con displasia severa o COCE fueron más fácilmente identificables con la quimioluminiscencia.

Con el azul de toluidina se tiñeron el 32,56\% de las lesiones no displásicas, el $58,82 \%$ de las lesiones con displasia leve o moderada y el $100 \%$ de las lesiones con displasia severa o carcinoma, con lo que presenta una sensibilidad y un VPN del $100 \%$ para lesiones con displasia severa y carcinoma. En cuan- to a los falsos positivos también son muy elevados, ya que el $60 \%$ de las candidiasis y el $63 \%$ de las hiperplasias epiteliales retuvieron la tinción.

Los autores concluyen que la quimioluminiscencia facilita la visualización de lesiones, ya que mejora su brillo, color y textura, haciéndolas más identificables y aumentando así la sensibilidad de la exploración. El azul de toluidina además de tener una sensibilidad del $100 \%$ para las lesiones de cáncer, reduce el número de falsos positivos del dispositivo de quimioluminiscencia. El problema de este estudio es que está realizado con pacientes de riesgo y por expertos en medicina bucal, por lo que no se puede extrapolar a la población general. Hay que tener en consideración que este estudio es el único que presenta conflicto de interés, ya que los autores trabajan para la marca comercial, siendo ésta también la que financia el estudio sobre uno de sus productos.

Otros autores, Oh y Laskin (36), seleccionaron 100 pacientes de riesgo libres de enfermedad para testar el dispositivo de quimioluminiscencia como método de screening. Observaron a los pacientes primero con la luz del sillón dental y exploración visual convencional, luego con la luz del sillón dental tras un enjuague de ácido acético y para terminar observaron la cavidad oral de los pacientes con el sistema de quimioluminiscencia. Localizaron un $90 \%$ de las lesiones con la exploración visual convencional y un $10 \%$ con la exploración bajo luz del sillón dental previo enjuague con ácido acético. No se halló ninguna lesión nueva con el sistema de quimioluminiscencia. Las lesiones identificadas fueron separadas en 2 grupos, las diagnosticables a primera vista, aquellas que pueden ser diagnosticadas clínicamente, (leucoedema, línea alba, mucosa masticada.) y las no diagnosticables a primera vista, aquellas en las que no es posible emitir un diagnóstico sólo con la exploración clínica. Estas últimas fueron sometidas a un análisis con OralCDx ${ }^{\mathrm{TM}}$, un sistema de citología por raspado o barrido ("brush biopsy") asistido por ordenador, el cual dividió a las muestras en "sospechosas de malignidad" y "no sospechosas de malignidad". Las muestras sospechosas fueron biopsiadas con bisturí frío, dando resultados negativos de malignidad. Los autores concluyen que la mejor manera para visua- 
lizar lesiones premalignas o cancerosas es la exploración visual con luz del sillón dental y que un enjuague previo con ácido acético puede ayudar, pero consideran que la exploración con luz química no sólo no ayuda sino que dificulta la exploración por la cantidad de reflejos que provoca.

En un estudio más reciente (42), se aplicó el sistema de quimioluminiscencia en 102 pacientes con lesiones clasificadas de inocuas por tres expertos en medicina bucal mediante una exploración visual convencional. Todas las lesiones fueron sometidas al método de luz química y posteriormente biopsiadas. Tres lesiones fueron definidas por los análisis anatomopatológicos con diferentes grados de displasia y una como carcinoma de células escamonas. Ninguna de las cuatro había resultado positiva al sistema de quimioluminiscencia. Por otro lado, 98 lesiones fueron definidas por el estudio anatomopatológico como con ningún signo de malignidad, 74 de ellas dieron negativo al test de quimioluminiscencia y 24 dieron positivo. Además no se halló ninguna lesión nueva al aplicar el sistema de luz química. Según este estudio la sensibilidad de la prueba sería del $0 \%$, la especificidad del 75,5\%, el VPP del $0 \%$ y el VPN del $94,8 \%$. La conclusión de los autores es que el dispositivo de quimioluminiscencia es completamente inútil ya que no detecta las lesiones malignas y además no detecta ninguna lesión que no se hubiera detectado bajo una exploración visual convencional. En este estudio los observadores vuelven a ser especialistas en medicina bucal y vuelven a utilizar el sistema de luz química como prueba diagnóstica y no como método de screening.

\section{CONCLUSIONES}

En nuestra revisión sólo encontramos ocho estudios que hayan probado un sistema de quimioluminiscencia como prueba de screening y diagnóstico. En la mayoría se ha utilizado el método como prueba diagnóstica y no como prueba de screening. En todos los casos los observadores fueron especialistas en medicina bucal y casi todos los pacientes observados ya presentaban una lesión en el momento de la prueba o eran pacientes de alto riesgo. Algunos estudios ponen de manifiesto que la utilización de quimioluminiscencia como método de screening de cáncer o precáncer oral aporta un ligero beneficio a la hora de observar las lesiones, mostrándolas más nítidas y claras, y en ocasiones mostrando lesiones no perceptibles a simple vista. Todos los autores coinciden al afirmar que este método no es capaz de distinguir las lesiones de carácter maligno de aquellas de carácter benigno, presentando por tanto unos valores de especificidad realmente bajos. Por otro lado, la opinión de algunos autores es que la prueba de quimioluminiscencia no supone ninguna ventaja respecto a la exploración convencional, mientras que para otros su uso se justifica por el hecho de ser capaz de localizar alguna lesión que bajo la luz del sillón no se aprecia.

Está claro que el método de la quimioluminiscencia no es una buena herramienta como prueba diagnóstica, es decir como prueba comparable a la biopsia, pero dadas las tasas de sensibilidad de la prueba se necesitarían más ensayos para concluir que ésta es una mala prueba de screening. En otros campos de la oncología desde hace tiempo se llevan utilizando pruebas de screening, con unos valores semejantes de sensibilidad, disminuyendo la mortalidad y morbilidad de la enfermedad y presentando buenos resultados a largo plazo respecto al coste-beneficio de la prueba. Quizás la diferencia con respecto a otras mucosas sea que la mucosa oral requiere de una interpretación por parte del clínico, ya que ésta está más expuesta a agentes externos de forma continua y por tanto las lesiones de tipo reactivo son muy frecuentes.

Casi todos los autores coinciden en que para llegar a unas conclusiones válidas respecto a la utilidad de la prueba serían necesarios un número superior de estudios, los cuales deberían cumplir unas condiciones extrapolables, como el tipo de observador y los criterios de inclusión de los pacientes, así como que la prueba fuera aplicada por dentistas generalistas, los pacientes estuvieran libres de enfermedad y el tiempo del estudio y los tamaños muestrales fueran realmente amplios.

\section{BIBLIOGRAFÍA}

1. IARC WHO. World cancer report. Geneve: Stewar BW; 2002. 
2. Mignogna MD, Fedele S, Lo Russo L. The World Cancer Report and the burden of oral cancer. Eur J Cancer Prev. 2004 Apr;13(2):139-42.

3 Sánchez MJ, Martinez C, Nieto A, Castellsagué $\mathrm{X}$, Quintana MJ, Bosch FX, et al. Oral and oropharyngeal cancer in Spain: Influence of dietary patterns. Eur J Cancer Prev. 2003 Feb;12(1):49-56.

4. Macfarlane GJ, Boyle P, Evstifeeva TV, Robertson C, Scully C. Rising trends of oral cancer mortality among males worldwide: The return of an old public health problem. Cancer Causes Control. 1994 May;5(3):259-65.

5. Robinson KL, Macfarlane GJ. Oropharyngeal cancer incidence and mortality in Scotland: are rates still increasing? Oral Oncol. 2003 Jan;39 (1):31-6.

6. Lingen MW, Kalmar JR, Karrison T, Speight PM. Critical evaluation of diagnostic aids for the detection of oral cancer. Oral Oncol. 2008 Jan; 44(1):10-22.

7. Epstein JB, Zhang L, Rosin M. Advances in the diagnosis of oral premalignant and malignant lesions. J Can Dent Assoc. 2002 Nov;68(10): 617-21.

8. Update January 1992: The American Cancer Society guidelines for the cancer-related checkup. CA Cancer J Clin. 1992 Jan-Feb;42(1):44-5.

9. Allison P, Franco E, Black M, Feine J. The role of professional diagnostic delays in the prognosis of upper aerodigestive tract carcinoma. Oral Oncol. 1998 Mar;34(2):147-53.

10. Kujan O, Duxbury AJ, Glenny AM, Thakker NS, Sloan P. Opinions and attitudes of the UK's GDPs and specialists in oral surgery, oral medicine and surgical dentistry on oral cancer screening. Oral Dis. 2006 Mar; 12(2):194-9.

11. Fedele S. Diagnostic aids in the screening of oral cancer. Head Neck Oncol. 2009 Jan 30;1 (1):5.
12. Jovanovic A, Kostense PJ, Schulten EA, Snow GB, van der Waal I. Delay in diagnosis of oral squamous cell carcinoma; a report from The Netherlands. Eur J Cancer B Oral Oncol. 1992 Jul;28B(1):37-8.

13. Williams PM, Poh CF, Hovan AJ, Ng S, Rosin MP. Evaluation of a suspicious oral mucosal lesion. J Can Dent Assoc. 2008 Apr;74(3):275-80.

14. Wilson JM, Jungner YG. Principles and practice of mass screening for disease. Bol Oficina Sanit Panam. 1968 Oct;65(4):281-393.

15. Ramadas K, Sankaranarayanan R, Jacob BJ, Thomas G, Somanathan T, Mahé C, et al. Interim results from a cluster randomized controlled oral cancer screening trial in kerala, India. Oral Oncol. 2003 Sep;39(6):580-8.

16. Blot WJ, McLaughlin JK, Winn DM, Austin DF, Greenberg RS, Preston-Martin S, et al. Smoking and drinking in relation to oral and pharyngeal cancer. Cancer Res. 1988 Jun 1;48(11):3282-7.

17. Shiboski CH, Shiboski SC, Silverman S Jr. Trends in oral cancer rates in the United States, 19731996. Community Dent Oral Epidemiol. 2000 Aug;28(4):249-56.

18. Speight PM, Palmer S, Moles DR, Downer MC, Smith DH, Henriksson M, et al. The costeffectiveness of screening for oral cancer in primary care. Health Technol Assess. 2006 Apr;10(14):1,144, iii-iv.

19. Sankaranarayanan R, Ramadas K, Thomas G, Muwonge R, Thara S, Mathew B, et al. Effect of screening on oral cancer mortality in Kerala, India: A cluster-randomised controlled trial. Lancet. 2005 Jun 4-10;365(9475):1927-33.

20. Mignogna MD, Fedele S. Oral cancer screening: 5 minutes to save a life. Lancet. 2005 Jun 410;365(9475):1905-6.

21. Downer MC, Jullien JA, Speight PM. An interim determination of health gain from oral cancer and precancer screening: 2 . Developing a model 
of population screening. Community Dent Health. 1997 Dec;14(4):227-32.

22. Sciubba JJ. Oral cancer and its detection. History-taking and the diagnostic phase of management. J Am Dent Assoc. 2001 Nov;132 Suppl:12S-8S.

23. Patton LL. The effectiveness of community-based visual screening and utility of adjunctive diagnostic aids in the early detection of oral cancer. Oral Oncol. 2003 Oct;39(7):708-23.

24. Jullien JA, Downer MC, Zakrzewska JM, Speight PM. Evaluation of a screening test for the early detection of oral cancer and precancer. Community Dent Health. 1995 Mar;12(1):3-7.

25. Horowitz AM, Alfano MC. Performing a deathdefying act. J Am Dent Assoc. 2001 Nov;132 Suppl:5S-6S.

26. Whited JD, Grichnik JM. The rational clinical examination. Does this patient have a mole or a melanoma? JAMA. 1998 Mar 4;279(9):696-701.

27. Shugars DC, Patton LL. Detecting, diagnosing, and preventing oral cancer. Nurse Pract. 1997 Jun;22(6):105, 109,10, 113-5 passim.

28. Burzynski NJ, Firriolo FJ, Butters JM, Sorrell CL. Evaluation of oral cancer screening. J Cancer Educ. 1997 Summer;12(2):95-9.

29. Thomson PJ. Field change and oral cancer: new evidence for widespread carcinogenesis? Int J Oral Maxillofac Surg. 2002 Jun;31(3):262-6.

30. Downer MC, Moles DR, Palmer S, Speight PM. A systematic review of test performance in screening for oral cancer and precancer. Oral Oncol. 2004 Mar;40(3):264-73.

31. Loiudice L, Abbiati R, Boselli F, Cecchini G, Costa S, Grossi E, et al. Improvement of Pap smear sensitivity using a visual adjunctive procedure: a co-operative Italian study on speculoscopy (GISPE). Eur J Cancer Prev. 1998 Aug;7(4):295304.
32. Mann W, Lonky N, Massad S, Scotti R, Blanco J, Vasilev S. Papanicolaou smear screening augmented by a magnified chemiluminescent exam. Int J Gynaecol Obstet. 1993 Dec;43(3): 289-96.

33. Massad LS, Lonky NM, Mutch DG, Mann WJ, Blanco JS, Vasilev SA, et al. Use of speculoscopy in the evaluation of women with atypical Papanicolaou smears. Improved cost effectiveness by selective colposcopy. J Reprod Med. 1993 Mar;38(3):163-9.

34. Farah CS, McCullough MJ. A pilot case control study on the efficacy of acetic acid wash and chemiluminescent illumination (ViziLite) in the visualisation of oral mucosal white lesions. Oral Oncol. 2007 Sep;43(8):820-4.

35. Bhalang K, Suesuwan A, Dhanuthai K, Sannikorn P, Luangjarmekorn L, Swasdison S. The application of acetic acid in the detection of oral squamous cell carcinoma. Oral Surg Oral Med Oral Pathol Oral Radiol Endod. 2008 Sep;106 (3):371-6.

36. Oh ES, Laskin DM. Efficacy of the ViziLite system in the identification of oral lesions. J Oral Maxillofac Surg. 2007 Mar;65(3):424-6.

37. Ram S, Siar $\mathrm{CH}$. Chemiluminescence as a diagnostic aid in the detection of oral cancer and potentially malignant epithelial lesions. Int J Oral Maxillofac Surg. 2005 Jul;34(5):521-7.

38. Huber MA, Bsoul SA, Terezhalmy GT. Acetic acid wash and chemiluminescent illumination as an adjunct to conventional oral soft tissue examination for the detection of dysplasia: A pilot study. Quintessence Int. 2004 May;35(5):378-84.

39. Kerr AR, Sirois DA, Epstein JB. Clinical evaluation of chemiluminescent lighting: An adjunct for oral mucosal examinations. J Clin Dent. 2006;17(3): 59-63.

40. Epstein JB, Gorsky M, Lonky S, Silverman S Jr, Epstein JD, Bride M. The efficacy of oral lumenoscopy (ViziLite) in visualizing oral muco- 
sal lesions. Spec Care Dentist. 2006 Jul-Aug;26 (4):171-4.

41. Epstein JB, Silverman S Jr, Epstein JD, Lonky SA, Bride MA. Analysis of oral lesion biopsies identified and evaluated by visual examination, chemiluminescence and toluidine blue. Oral Oncol. 2008 Jun;44(6):538-44.

42. Mehrotra R, Singh M, Thomas S, Nair P, Pandya $S$, Nigam NS, et al. A cross-sectional study evaluating chemiluminescence and auto- fluorescence in the detection of clinically innocuous precancerous and cancerous oral lesions. J Am Dent Assoc. 2010 Feb;141(2):151-6.

\section{CORRESPONDENCIA}

Antonio Carrera Torres

Brasil, 12, $3^{\circ} \mathrm{A}$

41013 Sevilla

Correo electrónico: antonio.carreratorres@yahoo.es 